\title{
Heat kernel estimates for the Dirichlet fractional Laplacian
}

Received March 15, 2008 and in revised form September 21, 2008

\begin{abstract}
We consider the fractional Laplacian $-(-\Delta)^{\alpha / 2}$ on an open subset in $\mathbb{R}^{d}$ with zero exterior condition. We establish sharp two-sided estimates for the heat kernel of such a Dirichlet fractional Laplacian in $C^{1,1}$ open sets. This heat kernel is also the transition density of a rotationally symmetric $\alpha$-stable process killed upon leaving a $C^{1,1}$ open set. Our results are the first sharp twosided estimates for the Dirichlet heat kernel of a non-local operator on open sets.
\end{abstract}

Keywords. Fractional Laplacian, symmetric $\alpha$-stable process, heat kernel, transition density, Green function, exit time, Lévy system, boundary Harnack inequality, parabolic Harnack inequality, intrinsic ultracontractivity

\section{Introduction}

Second order elliptic differential operators and diffusion processes take up, respectively, central places in the theory of partial differential equations (PDE) and in probability theory (see [18] and [19] for example). There are close relationships between these two subjects. For a large class of second order elliptic differential operators $\mathcal{L}$ on $\mathbb{R}^{d}$, there is a diffusion process $X$ in $\mathbb{R}^{d}$ associated with it so that $\mathcal{L}$ is the infinitesimal generator of $X$, and vice versa. The connection between $\mathcal{L}$ and $X$ can also be seen as follows. The fundamental solution $p(t, x, y)$ of $\partial_{t} u=\mathcal{L} u$ (also called the heat kernel of $\mathcal{L}$ ) is the transition density of $X$. Thus obtaining sharp two-sided estimates for $p(t, x, y)$ is a fundamental problem in both analysis and probability theory. In fact, two-sided heat kernel estimates for diffusions in $\mathbb{R}^{d}$ have a long history and many beautiful results have been established. See $[12,14]$ and the references therein. But, due to the complication near the boundary, two-sided estimates on the transition density of killed diffusions in a domain $D$ (equivalently, the Dirichlet heat kernel) have been established only recently. See [13, 14, 15] for

Z.-Q. Chen: Department of Mathematics, University of Washington, Seattle, WA 98195, USA; e-mail: zchen@math.washington.edu

P. Kim: Department of Mathematical Sciences, Seoul National University, Seoul 151-747, South Korea; e-mail: pkim@snu.ac.kr

R. Song: Department of Mathematics, University of Illinois, Urbana, IL 61801, USA; e-mail: rsong@math.uiuc.edu

Mathematics Subject Classification (2010): Primary 60J35, 47G20, 60J75; Secondary 47D07 
upper bound estimates and [27] for a lower bound estimate of the Dirichlet heat kernels in bounded $C^{1,1}$ domains.

Markov processes with discontinuous sample paths constitute an important family of stochastic processes in probability theory. Recently there has been intense interest in nonGaussian stable processes, due to their importance both in theory and in applications. It is well-known that (cf., e.g., Janicki and Weron [20], Samorodnitsky and Taqqu [24]) many physical and economic systems should be and in fact have been successfully modeled by non-Gaussian stable processes.

In this paper we always assume that $\alpha \in(0,2)$. A (rotationally) symmetric $\alpha$-stable process $X=\left\{X_{t}, t \geq 0, \mathbb{P}_{x}, x \in \mathbb{R}^{d}\right\}$ in $\mathbb{R}^{d}$ is a Lévy process such that

$$
\mathbb{E}_{x}\left[e^{i \xi \cdot\left(X_{t}-X_{0}\right)}\right]=e^{-t|\xi|^{\alpha}} \quad \text { for every } x \in \mathbb{R}^{d} \text { and } \xi \in \mathbb{R}^{d} \text {. }
$$

The infinitesimal generator of a symmetric $\alpha$-stable process $X$ in $\mathbb{R}^{d}$ is the fractional Laplacian $-(-\Delta)^{\alpha / 2}$, which is a prototype of nonlocal operators. The fractional Laplacian can be written in the form

$$
-(-\Delta)^{\alpha / 2} u(x)=c \lim _{\varepsilon \downarrow 0} \int_{\left\{y \in \mathbb{R}^{d}:|y-x|>\varepsilon\right\}}(u(y)-u(x)) \frac{d y}{|x-y|^{d+\alpha}}
$$

for some constant $c=c(d, \alpha)$. Recently there has also been interest from the theory of PDE (such as singular obstacle problems) to study such fractional Laplacians (see, for example, [3, 25] and the references therein). We will use $p(t, x, y)$ to denote the transition density of $X$ (or equivalently the heat kernel of the fractional Laplacian $-(-\Delta)^{\alpha / 2}$ ). It is well-known (see, e.g., [1, 8]) that

$$
p(t, x, y) \asymp t^{-d / \alpha} \wedge \frac{t}{|x-y|^{d+\alpha}} \quad \text { on }(0, \infty) \times \mathbb{R}^{d} \times \mathbb{R}^{d} .
$$

Here and in what follows, for two nonnegative functions $f$ and $g$, the notation $f \asymp g$ means that there are positive constants $c_{1}$ and $c_{2}$ such that $c_{1} g(x) \leq f(x) \leq c_{2} g(x)$ in the common domain of definition of $f$ and $g$. For $a, b \in \mathbb{R}, a \wedge \bar{b}:=\min \{a, b\}$ and $a \vee b:=\max \{a, b\}$. The Euclidean distance between $x$ and $y$ is denoted by $|x-y|$. We will use $B(x, r)$ to denote the open ball centered at $x \in \mathbb{R}^{d}$ with radius $r>0$.

For every open subset $D \subset \mathbb{R}^{d}$, we denote by $X^{D}$ the subprocess of $X$ killed upon leaving $D$. The infinitesimal generator of $X^{D}$ is the Dirichlet fractional Laplacian $-\left.(-\Delta)^{\alpha / 2}\right|_{D}$ (the fractional Laplacian with zero exterior condition). It is known (see [8]) that $X^{D}$ has a transition density $p_{D}(t, x, y)$ with respect to the Lebesgue measure that is jointly Hölder continuous. The connection between second order elliptic differential operators and diffusion processes can be extended to a large class of Markov processes. In particular, the transition density of $X^{D}$ is the fundamental solution of $\partial_{t} u=-\left.(-\Delta)^{\alpha / 2}\right|_{D} u$ (also called the heat kernel of the Dirichlet fractional Laplacian $\left.-\left.(-\Delta)^{\alpha / 2}\right|_{D}\right)$.

The purpose of this paper is to establish, in Theorem 1.1. two-sided sharp estimates on $p_{D}(t, x, y)$ for every $t>0$. To state this theorem, we first recall that an open set $D$ in $\mathbb{R}^{d}$ (when $d \geq 2$ ) is said to be a $C^{1,1}$ open set if there exist a localization radius $R_{0}>0$ and a constant $\Lambda_{0}>0$ such that for every $z \in \partial D$, there is a $C^{1,1}$-function $\phi=\phi_{z}: \mathbb{R}^{d-1} \rightarrow \mathbb{R}$ 
satisfying $\phi(0)=0, \nabla \phi(0)=(0, \ldots, 0),\|\nabla \phi\|_{\infty} \leq \Lambda_{0},|\nabla \phi(x)-\nabla \phi(z)| \leq \Lambda_{0}|x-z|$, and an orthonormal coordinate system $C S_{z}: y=\left(y_{1}, \ldots, y_{d-1}, y_{d}\right):=\left(\widetilde{y}, y_{d}\right)$ with origin at $z$ such that

$$
B\left(z, R_{0}\right) \cap D=B\left(0, R_{0}\right) \cap\left\{y: y_{d}>\phi(\widetilde{y})\right\},
$$

where the ball $B\left(0, R_{0}\right)$ on the right hand side is in the coordinate system $C S_{z}$. The pair $\left(R_{0}, \Lambda_{0}\right)$ is called the characteristics of the $C^{1,1}$ open set $D$. We remark that in some papers, $C^{1,1}$ open sets defined above are called uniform $C^{1,1}$ open sets as $\left(R_{0}, \Lambda_{0}\right)$ is universal for all $z \in \partial D$. For $x \in \mathbb{R}^{d}$, let $\delta_{\partial D}(x)$ denote the Euclidean distance between $x$ and $\partial D$. It is well-known that any $C^{1,1}$ open set $D$ satisfies both the uniform interior ball condition and the uniform exterior ball condition: there exists $r_{0}<R_{0}$ such that for every $x \in D$ with $\delta_{\partial D}(x)<r_{0}$ and $y \in \mathbb{R}^{d} \backslash \bar{D}$ with $\delta_{\partial D}(y)<r_{0}$, there are $z_{x}, z_{y} \in \partial D$ such that $\left|x-z_{x}\right|=\delta_{\partial D}(x),\left|y-z_{y}\right|=\delta_{\partial D}(y)$ and $B\left(x_{0}, r_{0}\right) \subset D$ and $B\left(y_{0}, r_{0}\right) \subset \mathbb{R}^{d} \backslash \bar{D}$ for $x_{0}=z_{x}+r_{0}\left(x-z_{x}\right) /\left|x-z_{x}\right|$ and $y_{0}=z_{y}+r_{0}\left(y-z_{y}\right) /\left|y-z_{y}\right|$. By a $C^{1,1}$ open set in $\mathbb{R}$ we mean an open set which can be written as the union of disjoint intervals so that the minimum of their lengths is positive and the minimum of the distances between them is also positive. Note that a $C^{1,1}$ open set can be unbounded and disconnected.

Theorem 1.1. Let $D$ be a $C^{1,1}$ open subset of $\mathbb{R}^{d}$ with $d \geq 1$ and $\delta_{D}(x)$ the Euclidean distance between $x$ and $D^{c}$.

(i) For every $T>0$, on $(0, T] \times D \times D$,

$$
p_{D}(t, x, y) \asymp\left(1 \wedge \frac{\delta_{D}(x)^{\alpha / 2}}{\sqrt{t}}\right)\left(1 \wedge \frac{\delta_{D}(y)^{\alpha / 2}}{\sqrt{t}}\right)\left(t^{-d / \alpha} \wedge \frac{t}{|x-y|^{d+\alpha}}\right) .
$$

(ii) Suppose in addition that $D$ is bounded. For every $T>0$, there are positive constants $c_{1}<c_{2}$ such that on $[T, \infty) \times D \times D$,

$$
c_{1} e^{-\lambda_{1} t} \delta_{D}(x)^{\alpha / 2} \delta_{D}(y)^{\alpha / 2} \leq p_{D}(t, x, y) \leq c_{2} e^{-\lambda_{1} t} \delta_{D}(x)^{\alpha / 2} \delta_{D}(y)^{\alpha / 2},
$$

where $\lambda_{1}>0$ is the smallest eigenvalue of the Dirichlet fractional Laplacian $\left.(-\Delta)^{\alpha / 2}\right|_{D}$.

By integrating the two-sided heat kernel estimates in Theorem 1.1 with respect to $t$, one can easily recover the following estimate on the Green function $G_{D}(x, y)=$ $\int_{0}^{\infty} p_{D}(t, x, y) d t$, initially obtained independently in [11] and [21] when $d \geq 2$.

Corollary 1.2. Let $D$ be a bounded $C^{1,1}$ open set in $\mathbb{R}^{d}$ with $d \geq 1$. Then on $D \times D$,

$$
G_{D}(x, y) \asymp \begin{cases}\frac{1}{|x-y|^{d-\alpha}}\left(1 \wedge \frac{\delta_{D}(x)^{\alpha / 2} \delta_{D}(y)^{\alpha / 2}}{|x-y|^{\alpha}}\right) & \text { when } d>\alpha, \\ \log \left(1+\frac{\delta_{D}(x)^{\alpha / 2} \delta_{D}(y)^{\alpha / 2}}{|x-y|^{\alpha}}\right) & \text { when } d=1=\alpha, \\ \left(\delta_{D}(x) \delta_{D}(y)\right)^{(\alpha-1) / 2} \wedge \frac{\delta_{D}(x)^{\alpha / 2} \delta_{D}(y)^{\alpha / 2}}{|x-y|} & \text { when } d=1<\alpha .\end{cases}
$$


Theorem 1.1(i) will be established through Theorems 2.4 and 3.1, which give the upper bound and lower bound estimates, respectively. Theorem 1.1.ii) is an easy consequence of the intrinsic ultracontractivity of a symmetric $\alpha$-stable process in a bounded $C^{1,1}$ open set. The latter will be reviewed and discussed in Section 3. In fact, the upper bound estimates in both Theorem 1.1 and Corollary 1.2 hold for any domain $D$ with (a weak version of) the uniform exterior ball condition in place of the $C^{1,1}$ condition, while the lower bound estimates in both Theorem 1.1 and Corollary 1.2 hold for any domain $D$ with the uniform interior ball condition in place of the $C^{1,1}$ condition (see Theorems 2.4 and 3.1 , and the proofs for Theorem 1.1.ii) and Corollary 1.2.

Although two-sided heat kernel estimates for jump processes in $\mathbb{R}^{d}$ have been studied recently by several authors (see [8, 9, 5] and the references therein), as far as we know, this is the first time that sharp two-sided estimates on the Dirichlet heat kernels for jump processes in open sets are established. We point out that, in addition to the use of the twosided estimate (1.1) of $p(t, x, y)$, the stable-scaling of $X$ and the Lévy system of $X$ and the boundary Harnack principle of $X$ in the annulus $U:=\left\{x \in \mathbb{R}^{d}: a<|x|<b\right\}$, only the following exit time estimate is used to get the upper bound estimate for $p_{D}(t, x, y)$ for $t \leq T$ :

$$
\mathbb{E}_{x}\left[\tau_{U}\right] \leq c_{\alpha, a, b} \delta_{U}(x)^{\alpha / 2} \quad \text { for } x \in U
$$

Here and in what follows, for any open set $D \subset \mathbb{R}^{d}, \tau_{D}:=\inf \left\{t>0: X_{t} \notin D\right\}$ denotes the first exit time from $D$ by $X$.

There are fundamental differences between obtaining two-sided Dirichlet heat kernel estimates for the Laplacian and the fractional Laplacian.

(i) Unlike the Dirichlet heat kernel for the Laplacian, the Dirichlet heat kernel for the fractional Laplacian does not have exponential decay in $|x-y|$. Thus we cannot use the chaining method, which is used to prove off-diagonal lower bound estimates of the Dirichlet heat kernel for the Laplacian.

(ii) Davies developed in [12] (see also [4, Section 3]) a very useful method to obtain off-diagonal upper bound estimates for the heat kernel of diffusions in the whole space. This method can also be used to prove off-diagonal upper bound estimates for the heat kernel of the Dirichlet Laplacian. Unfortunately we are unable to apply this powerful method to obtain upper bound estimates for the heat kernel of the Dirichlet fractional Laplacian.

(iii) In [27], a scale invariant parabolic boundary Harnack inequality obtained in [16] is used to obtain a sharp lower bound heat kernel estimate for the Dirichlet Laplacian. Such a parabolic boundary Harnack inequality is not available for the fractional Laplacian.

Due to the above differences and difficulties, obtaining two-sided sharp estimates on $p_{D}(t, x, y)$ for the fractional Laplacian with zero exterior condition requires new ideas and approaches. Our approach is mainly probabilistic. It uses only the following five ingredients:

(i) the upper bound heat kernel estimate in 1.1 for the rotationally symmetric $\alpha$-stable process $X$ in $\mathbb{R}^{d}$ and the stable-scaling property of $X$ (see 3.1 below); 
(ii) the Lévy system of $X$ that describes how the process jumps (see (2.1p);

(iii) the mean exit time estimates (1.2) established in Lemma 2.1 and the two-sided estimates in the ball $B=B(0,1): \mathbb{E}_{x}\left[\tau_{B}\right] \asymp \delta_{B}(x)^{\alpha / 2}$;

(iv) the boundary Harnack inequality of $X$ in annuli (when $d \geq 2$ ) and in intervals (when $d=1$ ), and the parabolic Harnack inequality of $X$;

(v) the intrinsic ultracontractivity of $X$ in bounded open sets.

The upper bound heat kernel estimate in (1.1) gives an upper bound for $p_{D}(t, x, y)$, while the Lévy system is the basic tool used throughout our argument as the symmetric stable process moves by "pure jumping". To get the boundary decay rate of $p_{D}(t, x, y)$, we use the boundary Harnack inequality and the domain monotonicity of the killed stable process $X^{D}$ in $D$ by comparing it with certain truncated exterior balls (i.e. annuli) as well as interior balls. The mean exit time estimate (1.2) established in Lemma 2.1] for an annulus with the help of the boundary Harnack inequality is applied to get the boundary decay rate in the upper bound heat kernel estimates in Lemma 2.2 and Theorem 2.3 . The two-sided estimate in the ball $B=B(0,1), \mathbb{E}_{x}\left[\tau_{B}\right] \asymp \delta_{B}(x)^{\alpha / 2}$, is used to get the twosided estimate (3.10) on the first eigenfunction in balls. The latter is then used in the proof of Lemma 3.6 to get the boundary decay rate for the lower bound estimate in $p_{D}(t, x, y)$. The parabolic Harnack inequality allows us to get a pointwise lower bound on $p_{D}(t, x, y)$ from the integral of $w \mapsto p_{D}(t / 2, x, w)$ over a suitable region, which is used in the proof of Proposition 3.3 When $X^{D}$ is intrinsic ultracontractive, $p_{D}(t, x, y)$ is comparable to $c_{t} \phi_{D}(x) \phi_{D}(y)$ for some $c_{t}>0$ and a good control is known for $c_{t}$ when $t$ is above a certain large $t_{0}$, where $\phi_{D}$ is the positive first eigenfunction of $\left.(-\Delta)^{\alpha / 2}\right|_{D}$. This property is used in the proof of Lemma 3.6 for balls and in the proof of Theorem 1.1 (ii).

The ideas developed in this paper can be used to study heat kernel estimates for other types of jump processes in open subsets and their perturbations. In fact, in [6] and [7] the ideas of this paper have been adapted and further developed to obtain two-sided sharp estimates for the transition density of censored stable processes and relativistic stable processes in $C^{1,1}$ open sets, respectively.

Throughout this paper, $d \geq 1$. We use $c_{1}, c_{2}, \ldots$ to denote generic constants, whose exact values are not important and can change from one appearance to another. The labeling of the constants $c_{1}, c_{2}, \ldots$ starts anew in the statement of each result. The dependence of the constant $c$ on the dimension $d$ will not be mentioned explicitly. We will use " $==$ " to denote a definition, which is read as "is defined to be". We will use $\partial$ to denote a cemetery point and for every function $f$, we extend its definition to $\partial$ by setting $f(\partial)=0$. We let $d x$ denote the Lebesgue measure in $\mathbb{R}^{d}$. For a Borel set $A \subset \mathbb{R}^{d}$, we also use $|A|$ to denote its Lebesgue measure.

\section{Upper bound estimate}

Throughout this section we assume that $D$ is an open set satisfying the uniform exterior ball condition with radius $r_{0}>0$ in the following sense: for every $z \in \partial D$ and $r \in\left(0, r_{0}\right)$, there is a ball $B^{z}$ of radius $r$ such that $B^{z} \subset \mathbb{R}^{d} \backslash \bar{D}$ and $\partial B^{z} \cap \partial D=\{z\}$. The goal of this section is to establish an upper bound for the transition density (heat kernel) $p_{D}(t, x, y)$. 
It is well-known that the symmetric stable process $X$ has Lévy intensity function

$$
J(x, y)=\mathcal{A}(d,-\alpha)|x-y|^{-(d+\alpha)},
$$

where

$$
\mathcal{A}(d,-\alpha)=\frac{\alpha \Gamma((d+\alpha) / 2)}{2^{1-\alpha} \pi^{d / 2} \Gamma(1-\alpha / 2)} .
$$

Here $\Gamma$ is the Gamma function defined by $\Gamma(\lambda):=\int_{0}^{\infty} t^{\lambda-1} e^{-t} d t$ for every $\lambda>0$. The Lévy intensity function gives the Lévy system for $X$, which describes the jumps of the process $X$ : for any nonnegative measurable function $f$ on $\mathbb{R}_{+} \times \mathbb{R}^{d} \times \mathbb{R}^{d}$ with $f(s, y, y)=0$ for all $s>0$ and $y \in \mathbb{R}^{d}$, for any $x \in \mathbb{R}^{d}$ and any stopping time $T$ (with respect to the filtration of $X$ ),

$$
\mathbb{E}_{x}\left[\sum_{s \leq T} f\left(s, X_{s-}, X_{S}\right)\right]=\mathbb{E}_{x}\left[\int_{0}^{T}\left(\int_{\mathbb{R}^{d}} f\left(s, X_{s}, y\right) J\left(X_{s}, y\right) d y\right) d s\right] .
$$

(See, for example, [8, proof of Lemma 4.7] and [9, Appendix A].)

In several places in this paper including the next lemma, we will use the following well-known fact (see [17]): for every $d \geq 1$ and $\alpha \in(0,2)$, there exists $c=c(\alpha)>0$ such that for every $x_{0} \in \mathbb{R}^{d}$ and $r>0$,

$$
\mathbb{E}_{x}\left[\tau_{B\left(x_{0}, r\right)}\right]=c\left(r^{2}-\left|x-x_{0}\right|^{2}\right)^{\alpha / 2} \quad \text { for } x \in B\left(x_{0}, r\right) .
$$

Lemma 2.1. Let $U:=\left\{z \in \mathbb{R}^{d}: r_{0}<|z|<3 r_{0} / 2\right\}$. There is a constantc $=c\left(r_{0}, \alpha\right)>0$ such that

$$
\mathbb{E}_{x}\left[\tau_{U}\right] \leq c \delta_{U}(x)^{\alpha / 2} \quad \text { for } r_{0}<|x|<5 r_{0} / 4 .
$$

Proof. For $d \geq 2$, we use the upper bound of the Green function $G_{U}(x, y)$ from (1.4) in [11, Theorem 1.1] (cf. also [21]) to deduce that $\mathbb{E}_{x}\left[\tau_{U}\right] \leq c \delta_{U}(x)^{\alpha / 2}$.

Now let $d=1$ and, without loss of generality, assume $x>0$. Let $B$ be the open interval $\left(r_{0}, 3 r_{0} / 2\right)$. Note that $\delta_{B}(x)=\delta_{U}(x)$. Taking $x_{0}=9 r_{0} / 8$, by the Lévy system 2.1] with $f(s, x, y)=\mathbf{1}_{U}(x) \mathbf{1}_{\left\{5 r_{0}<|y|<10 r_{0}\right\}}(y)$ and $T=\tau_{U}$, we have, on $U$,

$$
\mathbb{P}_{x}\left(10 r_{0}>\left|X_{\tau_{U}}\right|>5 r_{0}\right)=\mathbb{E}_{x}\left[\int_{0}^{\tau_{U}} \int_{\left\{5 r_{0}<|y|<10 r_{0}\right\}} \frac{\mathcal{A}(d,-\alpha)}{\left|X_{s}-y\right|^{d+\alpha}} d y d s\right] \asymp \mathbb{E}_{x}\left[\tau_{U}\right] .
$$

Hence by the boundary Harnack principle for $X$ in $B$ (see [2, Remark 6]),

$$
\begin{aligned}
\mathbb{E}_{x}\left[\tau_{U}\right] & \leq c_{1} \mathbb{P}_{x}\left(10 r_{0}>\left|X_{\tau_{B}}\right|>5 r_{0}\right) \frac{\mathbb{P}_{x_{0}}\left(10 r_{0}>\left|X_{\tau_{U}}\right|>5 r_{0}\right)}{\mathbb{P}_{x_{0}}\left(10 r_{0}>\left|X_{\tau_{B}}\right|>5 r_{0}\right)} \\
& \leq c_{2} \mathbb{P}_{x}\left(10 r_{0}>\left|X_{\tau_{B}}\right|>5 r_{0}\right)
\end{aligned}
$$

for some positive constants $c_{i}=c_{i}\left(r_{0}, \alpha\right), i=1,2$. By 2.2 and 2.3 but with $B$ in place of $U$, we conclude that

$$
\mathbb{E}_{x}\left[\tau_{U}\right] \leq c_{3} \mathbb{E}_{x}\left[\tau_{B}\right] \leq c_{4} \delta_{B}(x)^{\alpha / 2}=c_{4} \delta_{U}(x)^{\alpha / 2}
$$

for some positive constants $c_{i}=c_{i}\left(r_{0}, \alpha\right), i=3,4$. 
Lemma 2.2. Let $D=\left\{x \in \mathbb{R}^{d}:|x|>r_{0}\right\}$. For every $T>0$, there is a constant $c=c\left(r_{0}, \alpha\right)>0$ such that

$$
p_{D}(t, x, y) \leq c(T \vee 1) \frac{\delta_{D}(x)^{\alpha / 2}}{|x-y|^{d+\alpha}} \quad \text { for } r_{0}<|x|<5 r_{0} / 4,|y| \geq 2 r_{0} \text { and } t \leq T .
$$

Proof. Define $U:=\left\{z \in \mathbb{R}^{d}: r_{0}<|z|<3 r_{0} / 2\right\}$. It is well-known (see, e.g., [2]) that $X_{\tau_{U}} \notin \partial U$. For $r_{0}<|x|<5 r_{0} / 4,|y| \geq 2 r_{0}$ and $t \in(0, T]$, it follows from the strong Markov property and (2.1) that

$$
\begin{aligned}
& p_{D}(t, x, y)=\mathbb{E}_{x}\left[p_{D}\left(t-\tau_{U}, X_{\tau_{U}}, y\right): \tau_{U}<t\right] \\
&= \int_{0}^{t}\left(\int_{U} p_{U}(s, x, z)\left(\int_{\left\{w:|w|>3 r_{0} / 2\right\}} J(z, w) p_{D}(t-s, w, y) d w\right) d z\right) d s \\
&= \int_{0}^{t}\left(\int_{U} p_{U}(s, x, z)\left(\int_{\left\{w: 3 r_{0} / 4+|y| / 2 \geq|w|>3 r_{0} / 2\right\}} J(z, w) p_{D}(t-s, w, y) d w\right) d z\right) d s \\
& \quad+\int_{0}^{t}\left(\int_{U} p_{U}(s, x, z)\left(\int_{\left\{w:|w|>3 r_{0} / 4+|y| / 2\right\}} J(z, w) p_{D}(t-s, w, y) d w\right) d z\right) d s \\
&=: I+I I .
\end{aligned}
$$

Note that for $|w| \leq 3 r_{0} / 4+|y| / 2$,

$$
|w-y| \geq \frac{1}{2}\left(|y|-\frac{3 r_{0}}{2}\right) \geq \frac{|y|}{8} \geq \frac{|x-y|}{16} .
$$

Since $p_{D}(t-s, w, y) \leq p(t-s, w, y)$, by (1.1) and [2.4), there exist constants $c_{1}=$ $c_{1}(\alpha)>0$ and $c_{2}=c_{2}(\alpha)>0$ such that

$$
\begin{aligned}
I & \leq \int_{0}^{t}\left(\int_{U} p_{U}(s, x, z)\left(\int_{\left\{w: 3 r_{0} / 4+|y| / 2 \geq|w|>3 r_{0} / 2\right\}} J(z, w) \frac{c_{1} T}{|w-y|^{d+\alpha}} d w\right) d z\right) d s \\
& \leq \frac{c_{2} T}{|x-y|^{d+\alpha}} \int_{0}^{t}\left(\int_{U} p_{U}(s, x, z)\left(\int_{\left\{w: 3 r_{0} / 4+|y| / 2 \geq|w|>3 r_{0} / 2\right\}} J(z, w) d w\right) d z\right) d s \\
& =\frac{c_{2} T}{|x-y|^{d+\alpha}} \mathbb{P}_{x}\left(3 r_{0} / 2<\left|X_{\tau_{U}}\right| \leq 3 r_{0} / 4+|y| / 2 ; \tau_{U} \leq t\right) \\
& \leq \frac{c_{2} T}{|x-y|^{d+\alpha}} \mathbb{P}_{x}\left(\left|X_{\tau_{U}}\right|>3 r_{0} / 2\right) .
\end{aligned}
$$

Without loss of generality, assume that $x=\left(x_{1}, \ldots, x_{d}\right)$ with $x_{1}>0$. Taking $x_{0}=$ $\left(9 r_{0} / 8,0, \ldots, 0\right)$, by the boundary Harnack principle for $X$ in $U$ when $d \geq 2$ and in $B=\left(r_{0}, 3 r_{0} / 2\right)$ when $d=1$ (see Theorem 1 and Remark 6 of [2]), we have, for $|x| \in\left(r_{0}, 5 r_{0} / 4\right)$ with $x_{1}>0$,

$$
\begin{aligned}
\mathbb{P}_{x}\left(\left|X_{\tau_{U}}\right|>3 r_{0} / 2\right) & \leq c_{3} \mathbb{P}_{x}\left(10 r_{0}>\left|X_{\tau_{U}}\right|>5 r_{0}\right) \frac{\mathbb{P}_{x_{0}}\left(\left|X_{\tau_{U}}\right|>3 r_{0} / 2\right)}{\mathbb{P}_{x_{0}}\left(10 r_{0}>\left|X_{\tau_{U}}\right|>5 r_{0}\right)} \\
& \leq c_{4} \mathbb{P}_{x}\left(10 r_{0}>\left|X_{\tau_{U}}\right|>5 r_{0}\right) \\
& =c_{4} \mathbb{E}_{x}\left[\int_{0}^{\tau_{U}} \int_{\left\{5 r_{0}<|y|<10 r_{0}\right\}} \frac{\mathcal{A}(d,-\alpha)}{\left|X_{s}-y\right|^{d+\alpha}} d y d s\right] \leq c_{5} \mathbb{E}_{x}\left[\tau_{U}\right]
\end{aligned}
$$


for some positive constants $c_{i}=c_{i}\left(r_{0}, \alpha\right), i=3,4,5$. Thus by Lemma 2.1, we have

$$
I \leq c_{6}(T \vee 1) \frac{\delta_{D}(x)^{\alpha / 2}}{|x-y|^{d+\alpha}}
$$

for some positive constant $c_{6}=c_{6}\left(r_{0}, \alpha\right)$. On the other hand, for $z \in U$ and $w \in \mathbb{R}^{d}$ with $|w|>3 r_{0} / 4+|y| / 2$,

$$
|z-w| \geq \frac{1}{2}\left(|y|-\frac{3 r_{0}}{2}\right) \geq \frac{|y|}{8} \geq \frac{|x-y|}{16} .
$$

Thus by the symmetry of $p_{D}(t-s, w, y)$ in $(w, y)$, we have

$$
\begin{aligned}
I I & \leq \int_{0}^{t}\left(\int_{U} p_{U}(s, x, z)\left(\int_{\left\{w:|w|>3 r_{0} / 4+|y| / 2\right\}} \frac{c_{7}}{|x-y|^{d+\alpha}} p_{D}(t-s, y, w) d w\right) d z\right) d s \\
& \leq \frac{c_{7}}{|x-y|^{d+\alpha}} \int_{0}^{\infty}\left(\int_{U} p_{U}(s, x, z) d z\right) d s \\
& =\frac{c_{7}}{|x-y|^{d+\alpha}} \mathbb{E}_{x}\left[\tau_{U}\right] \leq \frac{c_{8} \delta_{D}(x)^{\alpha / 2}}{|x-y|^{d+\alpha}}
\end{aligned}
$$

for some positive constants $c_{k}=c_{k}\left(r_{0}, \alpha\right)$ for $k=7,8$. In the last inequality, we used Lemma 2.1 to deduce that $\mathbb{E}_{x}\left[\tau_{U}\right] \leq c \delta_{U}(x)^{\alpha / 2}=c \delta_{D}(x)^{\alpha / 2}$ for some positive constant $c=c\left(r_{0}, \alpha\right)$. This together with 2.5) proves the lemma.

A result similar to Lemma 2.2 has been established in [23, Theorem 4.2] for relativistic $\alpha$-stable processes. Our proof seems to be simpler. Moreover, unlike [23], we also cover the case $d=1$.

Theorem 2.3. Let $D$ be an open set that satisfies the uniform exterior ball condition with radius $r_{0}>0$. Then for every $T>0$, there is a constant $c=c\left(r_{0} / T, \alpha\right)>0$ independent of $\lambda \in(0, T]$ such that for $x, y \in \lambda^{-1} D$,

$$
p_{\lambda^{-1} D}(1, x, y) \leq c \min \left\{1,|x-y|^{-d-\alpha}\right\} \delta_{\lambda^{-1} D}(x)^{\alpha / 2} .
$$

Proof. Since for any open subset $U \subset \mathbb{R}^{d}$, by 1.1 ,

$$
p_{U}(t, x, y) \leq p(t, x, y) \asymp t^{-d / \alpha} \wedge \frac{t}{|x-y|^{d+\alpha}} \quad \text { on }(0, \infty) \times U \times U,
$$

it suffices to prove the conclusion for $x \in \lambda^{-1} D$ with $\delta_{\lambda^{-1} D}(x)<r_{0} /(4 T)$. Note that for every $\lambda \in(0, T], \lambda^{-1} D$ satisfies the uniform exterior ball condition with radius $r_{0} / T$. Let $z \in \partial\left(\lambda^{-1} D\right)$ with $|x-z|=\delta_{\lambda^{-1} D}(x)$. Let $B_{z}:=B\left(z_{0}, r_{0} / T\right) \subset\left(\lambda^{-1} D\right)^{c}$ be such that $\partial B_{z} \cap \partial\left(\lambda^{-1} D\right)=\{z\}$. When $\delta_{\lambda^{-1} D}(x)<r_{0} /(4 T)$ and $|x-y| \geq 5 r_{0} / T$, we have $\delta_{B_{z}^{c}}(y)>2 r_{0} / T$ and so by Lemma 2.2, there is a constant $c_{1}>0$ that depends only on $\left(r_{0} / T, d, \alpha\right)$ such that

$$
p_{\lambda^{-1} D}(t, x, y) \leq p_{\left(\bar{B}_{z}\right)^{c}}(t, x, y) \leq c_{1} \frac{\delta_{\left(\bar{B}_{z}\right)^{c}}(x)^{\alpha / 2}}{|x-y|^{d+\alpha}}=c_{1} \frac{\delta_{\lambda^{-1} D}(x)^{\alpha / 2}}{|x-y|^{d+\alpha}} \quad \text { for } t \leq 1 .
$$


So it remains to show that when $\delta_{\lambda^{-1} D}(x)<r_{0} /(4 T)$ and $|x-y|<5 r_{0} / T$, there exists a positive constant $c_{2}=c_{2}\left(r_{0} / T, d, \alpha\right)$ such that

$$
p_{\lambda^{-1} D}(1, x, y) \leq c_{2} \delta_{\lambda^{-1} D}(x)^{\alpha / 2} .
$$

The proof of (2.8) is similar to that of [26, Lemma 3.2], but for the readers' convenience we spell out the details. Define $U:=\left\{w \in \mathbb{R}^{d}:\left|w-z_{0}\right| \in\left(r_{0} / T, 8 r_{0} / T\right)\right\}$. Note that

$$
x, y \in U \cap \lambda^{-1} D \quad \text { and } \quad \delta_{U}(x)=\delta_{\lambda^{-1} D}(x) .
$$

By the strong Markov property and the symmetry of $p_{\lambda^{-1} D}(1, x, y)$ in $x$ and $y$, we have

$$
\begin{aligned}
p_{\lambda^{-1} D}(1, x, y)= & p_{U \cap \lambda^{-1} D}(1, x, y) \\
& +\mathbb{E}_{y}\left[p_{\lambda^{-1} D}\left(1-\tau_{U \cap \lambda^{-1} D}, X_{\tau_{U \cap \lambda^{-1} D}}, x\right) ; \tau_{U \cap \lambda^{-1} D}<\min \left\{1, \tau_{\lambda^{-1} D}\right\}\right] .
\end{aligned}
$$

By the semigroup property,

$$
\begin{aligned}
p_{U \cap \lambda^{-1} D}(1, x, y) & =\int_{U \cap \lambda^{-1} D} p_{U \cap \lambda^{-1} D}(1 / 2, x, z) p_{U \cap \lambda^{-1} D}(1 / 2, z, y) d z \\
& \leq\|p(1 / 2, \cdot, \cdot)\|_{\infty} \mathbb{P}_{x}\left(\tau_{U \cap \lambda^{-1} D}>1 / 2\right) \\
& \leq c_{3} \mathbb{E}_{x}\left[\tau_{U \cap \lambda^{-1} D}\right] \leq c_{3} \mathbb{E}_{x}\left[\tau_{U}\right] \leq c_{4} \delta_{U}(x)^{\alpha / 2}=c_{4} \delta_{\lambda^{-1} D}(x)^{\alpha / 2} .
\end{aligned}
$$

In the last inequality, we used Lemma 2.1 .

On the other hand, we have $X_{\tau_{U \cap \lambda^{-1} D}} \in U^{c} \cap \lambda^{-1} D$ on $\left\{\tau_{U \cap \lambda^{-1} D}<\min \left\{1, \tau_{\lambda^{-1} D}\right\}\right\}$, and so

$$
\left|X_{\tau_{U \cap \lambda^{-1} D}}-x\right| \geq 7 r_{0} / T \quad \text { on }\left\{\tau_{U \cap \lambda^{-1} D}<\min \left\{1, \tau_{\lambda^{-1} D}\right\}\right\} .
$$

Consequently, by 2.7 for $p_{\lambda^{-1} D}\left(1-\tau_{U \cap \lambda^{-1} D}, X_{\tau_{U \cap \lambda^{-1} D}}, x\right)$,

$$
\begin{aligned}
\mathbb{E}_{y}\left[p_{\lambda^{-1} D}(1-\right. & \left.\left.\tau_{U \cap \lambda^{-1} D}, X_{\tau_{U \cap \lambda^{-1} D}}, x\right) ; \tau_{U \cap \lambda^{-1} D}<\min \left\{1, \tau_{\lambda^{-1} D}\right\}\right] \\
& \leq \mathbb{E}_{y}\left[c_{1} \frac{\delta_{\lambda^{-1} D}(x)^{\alpha / 2}}{\left|X_{\tau_{U \cap \lambda^{-1} D}}-x\right|^{d+\alpha}} ; \tau_{U \cap \lambda^{-1} D}<\min \left\{1, \tau_{\lambda^{-1} D}\right\}\right] \\
& \leq c_{5} \delta_{\lambda^{-1} D}(x)^{\alpha / 2} \mathbb{P}_{y}\left(\tau_{U \cap \lambda^{-1} D}<\min \left\{1, \tau_{\lambda^{-1} D}\right\}\right) \leq c_{5} \delta_{\lambda^{-1} D}(x)^{\alpha / 2}
\end{aligned}
$$

This completes the proof of (2.8) and hence of the theorem.

Theorem 2.4. Let $D$ be an open set that satisfies the uniform exterior ball condition with radius $r_{0}>0$. For every $T>0$, there exists a positive constant $c=c\left(T, r_{0}, \alpha\right)$ such that for $t \in(0, T]$ and $x, y \in D$,

$$
p_{D}(t, x, y) \leq c\left(1 \wedge \frac{\delta_{D}(x)^{\alpha / 2}}{\sqrt{t}}\right)\left(1 \wedge \frac{\delta_{D}(y)^{\alpha / 2}}{\sqrt{t}}\right)\left(t^{-d / \alpha} \wedge \frac{t}{|x-y|^{d+\alpha}}\right) .
$$


Proof. Fix $T>0$. By Theorem 2.3 there exists a positive constant $c_{1}=c_{1}\left(T, r_{0}, \alpha\right)$ such that for every $\lambda \in\left(0, T^{1 / \alpha}\right]$,

$$
p_{\lambda^{-1} D}(1, x, y) \leq c_{1} \min \left\{1,|x-y|^{-d-\alpha}\right\} \delta_{\lambda^{-1} D}(x)^{\alpha / 2} .
$$

Thus for every $t \leq T$,

$$
\begin{aligned}
p_{D}(t, x, y) & =t^{-d / \alpha} p_{t^{-1 / \alpha} D}\left(1, t^{-1 / \alpha} x, t^{-1 / \alpha} y\right) \\
& \leq c_{1} t^{-d / \alpha} \min \left\{1,\left|t^{-1 / \alpha}(x-y)\right|^{-d-\alpha}\right\} \delta_{t^{-1 / \alpha} D}\left(t^{-1 / \alpha} x\right)^{\alpha / 2} \\
& \leq c_{1}\left(t^{-d / \alpha} \wedge \frac{t}{|x-y|^{d+\alpha}}\right) \frac{\delta_{D}(x)^{\alpha / 2}}{\sqrt{t}} \leq c_{2} p(t, x, y) \frac{\delta_{D}(x)^{\alpha / 2}}{\sqrt{t}} .
\end{aligned}
$$

By symmetry, the above inequality holds with the roles of $x$ and $y$ interchanged. The semigroup property for $t \leq T$ yields

$$
\begin{aligned}
p_{D}(t, x, y) & =\int_{D} p_{D}(t / 2, x, z) p_{D}(t / 2, z, y) d z \\
& \leq c_{3} \frac{\delta_{D}(x)^{\alpha / 2} \delta_{D}(y)^{\alpha / 2}}{t} \int_{D} p(t / 2, x, z) p(t / 2, z, y) d z \\
& \leq c_{3} \frac{\delta_{D}(x)^{\alpha / 2} \delta_{D}(y)^{\alpha / 2}}{t} p(t, x, y) .
\end{aligned}
$$

This proves the upper bound 2.9$)$ by noting that $(1 \wedge a)(1 \wedge b)=\min \{1, a, b, a b\}$ for $a, b>0$.

When $D$ is a semibounded convex domain, estimate [2.9] is given in [26, Theorem 1.6].

\section{Lower bound estimate}

Throughout this section except for Proposition 3.3. Lemma 3.4 and Proposition 3.5, the open set $D$ is assumed to satisfy the uniform interior ball condition with radius $r_{0}>0$ in the following sense: for every $x \in D$ with $\delta_{D}(x)<r_{0}$, there is $z_{x} \in \partial D$ such that $\left|x-z_{x}\right|=\delta_{D}(x)$ and $B\left(x_{0}, r_{0}\right) \subset D$ for $x_{0}:=z_{x}+r_{0}\left(x-z_{x}\right) /\left|x-z_{x}\right|$. Clearly, $\mathrm{a}$ (uniform) $C^{1,1}$ open set satisfies the uniform interior ball condition.

The goal of this section is to prove the following lower bound for the heat kernel $p_{D}(t, x, y)$.

Theorem 3.1. For every $T>0$ there exists a positive constant $c=c\left(r_{0}, \alpha, T\right)$ such that for all $(t, x, y) \in(0, T] \times D \times D$,

$$
p_{D}(t, x, y) \geq c\left(1 \wedge \frac{\delta_{D}(x)^{\alpha / 2}}{\sqrt{t}}\right)\left(1 \wedge \frac{\delta_{D}(y)^{\alpha / 2}}{\sqrt{t}}\right)\left(t^{-d / \alpha} \wedge \frac{t}{|x-y|^{d+\alpha}}\right) .
$$

We start with the following simple result which will be used later. 
Lemma 3.2. For any positive constants $c$ and $a$, there exists $c_{1}=c_{1}(c, a, \alpha)>0$ such that for every $z \in \mathbb{R}^{d}$ and $\lambda>0$,

$$
\inf _{\substack{y \in \mathbb{R}^{d} \\ y-z \mid \leq c \lambda^{1 / \alpha}}} \mathbb{P}_{y}\left(\tau_{B\left(z, 2 c \lambda^{1 / \alpha}\right)}>a \lambda\right) \geq c_{1}>0 .
$$

Proof. Without loss of generality, we may and do assume that $z=0$. By the stablescaling for symmetric $\alpha$-stable proceses:

$$
\left\{\lambda^{-1 / \alpha}\left(X_{\lambda t}-X_{0}\right), t \geq 0\right\} \text { has the same distribution as }\left\{X_{t}-X_{0}, t \geq 0\right\}
$$

for every $\lambda>0$, we have

$$
\begin{aligned}
\inf _{\substack{y \in \mathbb{R}^{d} \\
|y| \leq c \lambda^{1 / \alpha}}} \mathbb{P}_{y}\left(\tau_{B\left(0,2 c \lambda^{1 / \alpha}\right)}>a \lambda\right) & =\inf _{\substack{y \in \mathbb{R}^{d} \\
|y| \leq c}} \mathbb{P}_{y}\left(\tau_{B(0,2 c)}>a\right) \\
& \geq \inf _{\substack{y \in \mathbb{R}^{d} \\
|y| \leq c}} \int_{B(y, c)} p_{B(y, c)}(a, y, u) d u \\
& =\int_{B(0, c)} p_{B(0, c)}(a, 0, u) d u .
\end{aligned}
$$

This proves the lemma.

We will first establish the conclusion of Theorem 3.1 for small $T$, that is, we will first assume that

$$
t \leq T_{0}:=\left(r_{0} / 16\right)^{\alpha} .
$$

For this, we need some preparation. Note that Proposition 3.3 , Lemma 3.4 and Proposition 3.5 below hold for any open set $D$ and for every $t>0$.

Proposition 3.3. Suppose that $(t, x, y) \in(0, \infty) \times D \times D$ with $\delta_{D}(x) \geq t^{1 / \alpha} \geq 2|x-y|$. Then there exists a positive constant $c=c(\alpha)$ such that

$$
p_{D}(t, x, y) \geq c t^{-d / \alpha} \text {. }
$$

Proof. Let $t \in(0, \infty)$ and $x, y \in D$ with $\delta_{D}(x) \geq t^{1 / \alpha} \geq 2|x-y|$. By the parabolic Harnack principle in [8, Proposition 4.3],

$$
p_{D}(t / 2, x, w) \leq c_{1} p_{D}(t, x, y) \quad \text { for } w \in B\left(x, 2 t^{1 / \alpha} / 3\right),
$$

where the constant $c_{1}>0$ is independent of $x, y$ and $t$. This together with Lemma 3.2 yields

$$
\begin{aligned}
p_{D}(t, x, y) & \geq \frac{1}{c_{1}\left|B\left(x, t^{1 / \alpha} / 2\right)\right|} \int_{B\left(x, t^{1 / \alpha} / 2\right)} p_{D}(t / 2, x, w) d w \\
& \geq c_{2} t^{-d / \alpha} \int_{B\left(x, t^{1 / \alpha} / 2\right)} p_{B\left(x, t^{1 / \alpha} / 2\right)}(t / 2, x, w) d w \\
& =c_{2} t^{-d / \alpha} \mathbb{P}_{x}\left(\tau_{B\left(x, t^{1 / \alpha} / 2\right)}>t / 2\right) \geq c_{3} t^{-d / \alpha},
\end{aligned}
$$

where $c_{i}=c_{i}(\alpha)>0$ for $i=2,3$. 
Lemma 3.4. Suppose that $(t, x, y) \in(0, \infty) \times D \times D$ with $\min \left\{\delta_{D}(x), \delta_{D}(y)\right\} \geq t^{1 / \alpha}$ and $|x-y| \geq 2^{-1} t^{1 / \alpha}$. Then there exists a constant $c=c(\alpha)>0$ such that

$$
\mathbb{P}_{x}\left(X_{t}^{D} \in B\left(y, 2^{-1} t^{1 / \alpha}\right)\right) \geq c \frac{t^{d / \alpha+1}}{|x-y|^{d+\alpha}} .
$$

Proof. The proof is a simple modification of that of Proposition 4.11 in [9]. For the readers' convenience, we spell out the details.

By Lemma 3.2. starting at $z \in B\left(y, 4^{-1} t^{1 / \alpha}\right)$, with probability at least $c_{1}=c_{1}(\alpha)>0$ the process $X$ does not move more than $6^{-1} t^{1 / \alpha}$ by time $t$. Thus, it is sufficient to show that there exists a constant $c_{2}=c_{2}(\alpha)>0$ such that

$$
\mathbb{P}_{x}\left(X^{D} \text { hits the ball } B\left(y, 4^{-1} t^{1 / \alpha}\right) \text { by time } t\right) \geq c_{2} \frac{t^{d / \alpha+1}}{|x-y|^{d+\alpha}}
$$

for all $|x-y| \geq 2^{-1} t^{1 / \alpha}$ and $t>0$. Now with $B_{x}:=B\left(x, 6^{-1} t^{1 / \alpha}\right), B_{y}:=B\left(y, 6^{-1} t^{1 / \alpha}\right)$ and $\tau_{x}:=\tau_{B_{x}}$, it follows from Lemma 3.2 that there exists $c_{3}=c_{3}(\alpha)>0$ such that

$$
\mathbb{E}_{x}\left[t \wedge \tau_{x}\right] \geq \frac{t}{2} \mathbb{P}_{x}\left(\tau_{x} \geq t / 2\right) \geq c_{3} t \quad \text { for } t>0 .
$$

Thus by using the Lévy system,

$$
\begin{aligned}
\mathbb{P}_{x}\left(X^{D} \text { hits the ball } B\left(y, 4^{-1} t^{1 / \alpha}\right) \text { by time } t\right) \\
\quad \geq \mathbb{P}_{x}\left(X_{t \wedge \tau_{x}} \in B\left(y, 4^{-1} t^{1 / \alpha}\right) \text { and } t \wedge \tau_{x} \text { is a jumping time }\right) \\
\quad \geq \mathbb{E}_{x}\left[\int_{0}^{t \wedge \tau_{x}} \int_{B_{y}} \frac{c_{4}}{\left|X_{s}-u\right|^{d+\alpha}} d u d s\right] \\
\quad \geq c_{5} \mathbb{E}_{x}\left[t \wedge \tau_{x}\right] \int_{B_{y}} \frac{1}{|x-y|^{d+\alpha}} d u \geq c_{6} t\left|B_{y}\right||x-y|^{-d-\alpha} \geq c_{7} \frac{t^{d / \alpha+1}}{|x-y|^{d+\alpha}}
\end{aligned}
$$

for some positive constants $c_{i}=c_{i}(\alpha), i=4,5,6,7$. Here in the fourth inequality, 3.5 is used. This establishes the lemma.

Proposition 3.5. Suppose that $(t, x, y) \in(0, \infty) \times D \times D$ with $\min \left\{\delta_{D}(x), \delta_{D}(y)\right\} \geq$ $(t / 2)^{1 / \alpha}$ and $|x-y| \geq 2^{-1}(t / 2)^{1 / \alpha}$. Then there exists a constant $c=c(\alpha)>0$ such that

$$
p_{D}(t, x, y) \geq c \frac{t}{|x-y|^{d+\alpha}} .
$$

Proof. By the semigroup property, Proposition 3.3 and Lemma 3.4 , there exist positive constants $c_{1}=c_{1}(\alpha)$ and $c_{2}=c_{2}(\alpha)$ such that

$$
\begin{aligned}
p_{D}(t, x, y) & =\int_{D} p_{D}(t / 2, x, z) p_{D}(t / 2, z, y) d z \\
& \geq \int_{B\left(y, 2^{-1}(t / 2)^{1 / \alpha}\right)} p_{D}(t / 2, x, z) p_{D}(t / 2, z, y) d z \\
& \geq c_{1} t^{-d / \alpha} \mathbb{P}_{x}\left(X_{t / 2}^{D} \in B\left(y, 2^{-1}(t / 2)^{1 / \alpha}\right)\right) \geq c_{2} \frac{t}{|x-y|^{d+\alpha}} .
\end{aligned}
$$


The proof of the next lemma uses the intrinsic ultracontractivity of $X$ in the ball $B(0,3)$. Recall that when an open set $U$ is bounded, the transition semigroup $\left\{P_{t}^{U}, t>0\right\}$ of the symmetric $\alpha$-stable process $X^{U}$, which is a strongly continuous contraction semigroup in $L^{2}(U, d x)$, is compact. Let $\lambda_{1}^{U}>0$ be the smallest eigenvalue of $\left.(-\Delta)^{\alpha / 2}\right|_{U}$ and let $\phi_{U}(x)$ be the positive eigenfunction of $P_{1}^{U}$ corresponding to $e^{-\lambda_{1}^{U}}$ with $\left\|\phi_{U}\right\|_{L^{2}(U)}=1$. The semigroup $\left\{P_{t}^{U}, t>0\right\}$ of $X^{U}$ in the bounded open set $U$ is said to be intrinsic ultracontractive if for any $t>0$ there are positive constants $c_{t}>1$ such that

$$
c_{t}^{-1} \phi_{U}(x) \phi_{U}(y) \leq p_{U}(t, x, y) \leq c_{t} \phi_{U}(x) \phi_{U}(y) \quad \text { for } x, y \in U .
$$

The notion of intrinsic ultracontactivity was introduced by Davies and Simon in [15]. It has many equivalent definitions (see [15, Theorem 3.2]). It follows from Theorem 4.2.5 of [14] that if $\left\{P_{t}^{U}, t>0\right\}$ is intrinsic ultracontractive, there exists $T_{1}>0$ such that for all $(t, x, y) \in\left[T_{1}, \infty\right) \times U \times U$,

$$
\frac{1}{2} e^{-\lambda_{1}^{U} t} \phi_{U}(x) \phi_{U}(y) \leq p_{U}(t, x, y) \leq \frac{3}{2} e^{-\lambda_{1}^{U} t} \phi_{U}(x) \phi_{U}(y) .
$$

It was shown in [10, Theorem 4.6] using the log-Sobolev inequality that for any bounded $C^{1,1}$ domain $U$, the semigroup $\left\{P_{t}^{U}, t>0\right\}$ of $X^{U}$ is intrinsic ultracontractive. It was later proved by Kulczycki [22] that the intrinsic ultracontractivity holds for the semigroup $\left\{P_{t}^{U}, t>0\right\}$ of $X^{U}$ in any bounded open set. Though in [22] it is assumed that $d \geq 2$, the proof there in fact works for $d=1$ as well. In particular the semigroup of $X^{B(0,3)}$ is intrinsic ultracontractive, and by [22, Theorem 9], there is a constant $c_{1}=c_{1}(\alpha)>0$ such that

$$
\begin{aligned}
\int_{B(0,1)} G_{B(0,3)}(x, y) d y & \geq c_{1} \int_{B(0,3)} G_{B(0,3)}(x, y) d y \\
& =c_{1} \mathbb{E}_{x}\left[\tau_{B(0,3)}\right] \text { for } x \in B(0,3) .
\end{aligned}
$$

The ground state $\phi_{B(0,3)}$ of $X^{B(0,3)}$ is bounded, strictly positive and continuous in $B(0,3)$. So by (2.2), for $x \in B(0,3)$,

$$
\begin{aligned}
\phi_{B(0,3)}(x) & =\lambda_{1}^{B(0,3)} G_{B(0,3)} \phi_{B(0,3)}(x) \leq \lambda_{1}^{B(0,3)}\left\|\phi_{B(0,3)}\right\|_{\infty} \mathbb{E}_{x}\left[\tau_{B(0,3)}\right] \\
& \leq c_{2} \delta_{B(0,3)}(x)^{\alpha / 2},
\end{aligned}
$$

while by 2.2 and 3.9),

$$
\begin{aligned}
\phi_{B(0,3)}(x) & =\lambda_{1}^{B(0,3)} G_{B(0,3)} \phi_{B(0,3)}(x) \geq c_{3} \int_{B(0,1)} G_{B(0,3)}(x, y) d y \geq c_{4} \mathbb{E}_{x}\left[\tau_{B(0,3)}\right] \\
& \geq c_{5} \delta_{B(0,3)}(x)^{\alpha / 2} .
\end{aligned}
$$

Here $\left\|\phi_{B(0,3)}\right\|_{\infty}:=\sup _{x \in B(0,3)} \phi_{B(0,3)}(x)$. In other words, we have

$$
\phi_{B(0,3)}(x) \asymp \delta_{B(0,3)}(x)^{\alpha / 2} \quad \text { on } B(0,3) .
$$


Lemma 3.6. Suppose that $(t, x) \in\left(0, T_{0}\right] \times D$ with $\delta_{D}(x)<3 t^{1 / \alpha}<r_{0}$ and $\kappa \in(0,1)$. There exists $z_{x} \in \partial D$ such that $\left|x-z_{x}\right|=\delta_{D}(x)$ and $B\left(x_{0}, r_{0}\right) \subset D$ where $x_{0}:=$ $z_{x}+r_{0}\left(x-z_{x}\right) /\left|x-z_{x}\right|$. Let $z_{0}=z_{x}+3 t^{1 / \alpha}\left(x-z_{x}\right) /\left|x-z_{x}\right|$. Suppose $B\left(x_{0}, 2 \kappa t^{1 / \alpha}\right) \subset$ $B\left(z_{0}, 3 t^{1 / \alpha}\right)$. Then for any $a>0$, there exists a constant $c=c(\kappa, \alpha, a)>0$ such that

$$
\mathbb{P}_{x}\left(X_{a t}^{D} \in B\left(x_{0}, \kappa t^{1 / \alpha}\right)\right) \geq c t^{-1 / 2} \delta_{D}(x)^{\alpha / 2} .
$$

Proof. The existence of $x_{0}$ follows from the definition of the uniform interior ball condition with radius $r_{0}$. For convenience, we may assume $z_{0}=0$ and let $\widehat{B}:=B\left(x_{0}, \kappa t^{1 / \alpha}\right)$ and $B:=B\left(0,3 t^{1 / \alpha}\right)$. Note that $x \in B \subset D$ and $\partial B \cap \partial D=\left\{z_{x}\right\}, t^{-1 / \alpha} B=B(0,3)$ and $t^{-1 / \alpha} \widehat{B}=B\left(t^{-1 / \alpha} x_{0}, \kappa\right)$ with $B\left(t^{-1 / \alpha} x_{0}, 2 \kappa\right) \subset B(0,3)$. By the stable-scaling [3.1,

$$
\begin{aligned}
\mathbb{P}_{x}\left(X_{a t}^{D} \in \widehat{B}\right) & =\mathbb{P}_{t^{-1 / \alpha} x}\left(X_{a}^{t^{-1 / \alpha} D} \in t^{-1 / \alpha} \widehat{B}\right) \\
& \geq \mathbb{P}_{t^{-1 / \alpha} x}\left(X_{a}^{t^{-1 / \alpha} B} \in t^{-1 / \alpha} \widehat{B}\right)=\mathbb{P}_{t^{-1 / \alpha} x}\left(X_{a}^{B(0,3)} \in t^{-1 / \alpha} \widehat{B}\right) .
\end{aligned}
$$

By 3.7) and 3.10, there is a constant $c_{1}=c_{1}(a, \alpha)>0$ such that

$$
p_{B(0,3)}(a, z, y) \geq c_{1} \delta_{B(0,3)}(z)^{\alpha / 2} \delta_{B(0,3)}(y)^{\alpha / 2} \quad \text { for } z, y \in B(0,3) .
$$

Hence we have

$$
\begin{aligned}
\mathbb{P}_{x}\left(X_{a t}^{D} \in \widehat{B}\right) & \geq c_{1} \delta_{B(0,3)}\left(t^{-1 / \alpha} x\right)^{\alpha / 2} \int_{t^{-1 / \alpha} \widehat{B}} \delta_{B(0,3)}(y)^{\alpha / 2} d y \\
& \geq c_{2} \delta_{t^{-1 / \alpha} D}\left(t^{-1 / \alpha} x\right)^{\alpha / 2}=c_{2} t^{-1 / 2} \delta_{D}(x)^{\alpha / 2}
\end{aligned}
$$

for some $c_{2}=c_{2}(\kappa, \alpha, a)>0$.

Proposition 3.7. Suppose that $(t, x, y) \in\left(0, T_{0}\right] \times D \times D$ with $|x-y| \leq t^{1 / \alpha}$ and $\delta_{D}(x) \leq 2 t^{1 / \alpha}$. Then there exists a constant $c=c\left(r_{0}, \alpha\right)>0$ such that

$$
p_{D}(t, x, y) \geq c t^{-d / \alpha-1} \delta_{D}(x)^{\alpha / 2} \delta_{D}(y)^{\alpha / 2} .
$$

Proof. Note that under the assumptions of the proposition, we have

$$
\delta_{D}(y) \leq|x-y|+\delta_{D}(x) \leq 3 t^{1 / \alpha}<r_{0} / 5
$$

So there are points $z_{x}, z_{y} \in \partial D$ such that $\left|x-z_{x}\right|=\delta_{D}(x),\left|y-z_{y}\right|=\delta_{D}(y)$ and $B\left(x_{1}, r_{0}\right) \subset D, B\left(y_{1}, r_{0}\right) \subset D$ for $x_{1}:=z_{x}+r_{0}\left(x-z_{x}\right) /\left|x-z_{x}\right|$ and $y_{1}:=z_{y}+$ $r_{0}\left(y-z_{y}\right) /\left|y-z_{y}\right|$. Let

$$
x_{0}=z_{x}+4 t^{1 / \alpha}\left(x-z_{x}\right) /\left|x-z_{x}\right| \quad \text { and } \quad y_{0}=z_{y}+4 t^{1 / \alpha}\left(y-z_{y}\right) /\left|y-z_{y}\right| .
$$

Observe that

$$
\delta_{D}\left(x_{0}\right)=\delta_{D}\left(y_{0}\right)=4 t^{1 / \alpha} \quad \text { and } \quad\left|x-x_{0}\right|,\left|y-y_{0}\right| \in\left[t^{1 / \alpha}, 4 t^{1 / \alpha}\right) .
$$


By the semigroup property, with $B:=B\left(x_{0}, 4^{-1} t^{1 / \alpha}\right)$ and $\widetilde{B}:=B\left(y_{0}, 4^{-1} t^{1 / \alpha}\right)$,

$$
\begin{aligned}
p_{D}(t, x, y) & =\int_{D} p_{D}(t / 3, x, z) \int_{D} p_{D}(t / 3, z, w) p_{D}(t / 3, w, y) d w d z \\
& \geq \int_{B} p_{D}(t / 3, x, z) \int_{\widetilde{B}} p_{D}(t / 3, z, w) p_{D}(t / 3, w, y) d w d z \\
& \geq \inf _{(z, w) \in B \times \widetilde{B}} p_{D}(t / 3, z, w) \int_{B} p_{D}(t / 3, x, z) d z \int_{\widetilde{B}} p_{D}(t / 3, w, y) d w .
\end{aligned}
$$

Since for $z \in B$ and $w \in \widetilde{B}$,

$$
\delta_{D}(z) \geq \delta_{D}\left(x_{0}\right)-\left|x_{0}-z\right| \geq t^{1 / \alpha}, \quad \delta_{D}(w) \geq \delta_{D}\left(y_{0}\right)-\left|y_{0}-w\right| \geq t^{1 / \alpha}
$$

and

$$
|z-w| \leq\left|z-x_{0}\right|+\left|x_{0}-x\right|+|x-y|+\left|y-y_{0}\right|+\left|y_{0}-w\right|<10 t^{1 / \alpha},
$$

by combining Propositions 3.3 and 3.5 , we find that there exists $c_{1}=c_{1}\left(\alpha, r_{0}\right)>0$ such that

$$
\inf _{(z, w) \in B \times \widetilde{B}} p_{D}(t / 3, z, w) \geq c_{1} t^{-d / \alpha} .
$$

Since $\delta_{D}(x) \leq 2 t^{1 / \alpha}<r_{0} / 8$ and $\delta_{D}(y) \leq 3 t^{1 / \alpha}$, by Lemma 3.6 we have

$$
p_{D}(t, x, y) \geq c_{2} t^{-d / \alpha-1} \delta_{D}(x)^{\alpha / 2} \delta_{D}(y)^{\alpha / 2}
$$

for some positive constant $c_{2}=c_{2}\left(\alpha, r_{0}\right)$.

Proposition 3.8. Suppose that $(t, x, y) \in\left(0, T_{0}\right] \times D \times D$ with $\delta_{D}(x) \leq t^{1 / \alpha}$ and $(t / 2)^{1 / \alpha} \leq \delta_{D}(y)$ and $|x-y| \geq t^{1 / \alpha}$. Then there exists a constant $c=c\left(\alpha, r_{0}\right)>0$ such that

$$
p_{D}(t, x, y) \geq c \frac{t^{1 / 2} \delta_{D}(x)^{\alpha / 2}}{|x-y|^{d+\alpha}} .
$$

Proof. Since $D$ is an open set satisfying the uniform interior ball condition with radius $r_{0}$ and $\delta_{D}(x) \leq t^{1 / \alpha} \leq r_{0} / 16$, there is $z_{x} \in \partial D$ such that $\left|x-z_{x}\right|=\delta_{D}(x)$ and $B\left(x_{1}, r_{0}\right) \subset$ $D$ for $x_{1}:=z_{x}+r_{0}\left(x-z_{x}\right) /\left|x-z_{x}\right|$. Define $z_{0}=z_{x}+2 t^{1 / \alpha}\left(x-z_{x}\right) /\left|x-z_{x}\right|$ and choose $x_{0}$ in $B\left(z_{0}, 2 t^{1 / \alpha}\right)$ and $\kappa=\kappa(\alpha) \in(0,1)$ such that

$$
B\left(x_{0}, 2 \kappa t^{1 / \alpha}\right) \subset B\left(z_{0},\left(2-2^{-2 / \alpha}\right) t^{1 / \alpha}\right) \cap B\left(x,\left(1-2^{-1-2 / \alpha}\right) t^{1 / \alpha}\right) .
$$

Such a ball $B\left(x_{0}, 2 \kappa t^{1 / \alpha}\right)$ always exists because

$$
2<\left(2-2^{-1}\right)+\left(1-2^{-2}\right)<\left(2-2^{-2 / \alpha}\right)+\left(1-2^{-1-2 / \alpha}\right) .
$$

Note that

$$
\delta_{D}(z) \geq(t / 4)^{1 / \alpha} \quad \text { and } \quad|y-z| \geq 2^{-1}(t / 4)^{1 / \alpha} \quad \text { for every } z \in B\left(x_{0}, \kappa t^{1 / \alpha}\right) .
$$


On the other hand, for every $z \in B\left(x_{0}, \kappa t^{1 / \alpha}\right)$,

$$
|z-y| \leq|z-x|+|x-y| \leq\left(1-2^{-1-2 / \alpha}\right) t^{1 / \alpha}+|x-y|<2|x-y| .
$$

Thus by the semigroup property and Proposition 3.5 there exist positive constants $c_{1}, c_{2}$ and $c_{3}$ depending only on $\alpha$ and $r_{0}$ such that

$$
\begin{aligned}
p_{D}(t, x, y) & =\int_{D} p_{D}(t / 2, x, z) p_{D}(t / 2, z, y) d z \\
& \geq \int_{B\left(x_{0}, \kappa t^{1 / \alpha}\right)} p_{D}(t / 2, x, z) p_{D}(t / 2, z, y) d z \\
& \geq c_{1} \int_{B\left(x_{0}, \kappa t^{1 / \alpha}\right)} p_{D}(t / 2, x, z) \frac{t}{|z-y|^{d+\alpha}} d z \\
& \geq c_{2} \frac{t}{|x-y|^{d+\alpha}} \int_{B\left(x_{0}, \kappa t^{1 / \alpha}\right)} p_{D}(t / 2, x, z) d z \\
& =c_{3} \frac{t}{|x-y|^{d+\alpha}} \mathbb{P}_{x}\left(X_{t / 2}^{D} \in B\left(x_{0}, \kappa t^{1 / \alpha}\right)\right) .
\end{aligned}
$$

Applying Lemma 3.6, we arrive at the conclusion of the proposition.

Proposition 3.9. Suppose that $(t, x, y) \in\left(0, T_{0}\right] \times D \times D$ with

$$
\max \left\{\delta_{D}(x), \delta_{D}(y)\right\} \leq(t / 2)^{1 / \alpha} \leq|x-y| .
$$

Then there exists a constant $c=c\left(\alpha, r_{0}\right)>0$ such that

$$
p_{D}(t, x, y) \geq c \frac{\delta_{D}(x)^{\alpha / 2} \delta_{D}(y)^{\alpha / 2}}{|x-y|^{d+\alpha}} .
$$

Proof. As in the first paragraph of the proof of Proposition 3.7, let $z_{x} \in \partial D$ be such that $\left|x-z_{x}\right|=\delta_{D}(x)$ and $B\left(x_{1}, r_{0}\right) \subset D$ for $x_{1}:=z_{x}+r_{0}\left(x-z_{x}\right) /\left|x-z_{x}\right|$. Let $x_{0}=z_{x}+3 t^{1 / \alpha}\left(x-z_{x}\right) /\left|x-z_{x}\right|$. Then $\partial B\left(x_{0}, 3 t^{1 / \alpha}\right) \cap \partial D=\left\{z_{x}\right\}$. Let $\kappa:=1-2^{-1 / \alpha}$. Note that

$\delta_{D}(z) \geq 2(t / 2)^{1 / \alpha}$ and $|y-z| \geq \delta_{D}(z)-\delta_{D}(y) \geq(t / 2)^{1 / \alpha} \quad$ for every $z \in B\left(x_{0}, \kappa t^{1 / \alpha}\right)$.

Thus by the semigroup property, the symmetric property of $p_{D}(t, y, z)$, and Propositions 3.5 and 3.8 there exist positive constants $c_{1}$ and $c_{2}$ depending only on $\alpha$ and $r_{0}$ such that

$$
\begin{aligned}
p_{D}(t, x, y) & =\int_{D} p_{D}(t / 2, x, z) p_{D}(t / 2, z, y) d z \\
& \geq \int_{B\left(x_{0}, \kappa t^{1 / \alpha}\right)} p_{D}(t / 2, x, z) p_{D}(t / 2, z, y) d z \\
& \geq c_{1} \int_{B\left(x_{0}, \kappa t^{1 / \alpha}\right)} p_{D}(t / 2, x, z) \frac{t^{1 / 2} \delta_{D}(y)^{\alpha / 2}}{|z-y|^{d+\alpha}} d z
\end{aligned}
$$




$$
\begin{aligned}
& \geq c_{2} \frac{t^{1 / 2} \delta_{D}(y)^{\alpha / 2}}{|x-y|^{d+\alpha}} \int_{B\left(x_{0}, \kappa t^{1 / \alpha}\right)} p_{D}(t / 2, x, z) d z \\
& =c_{2} \frac{t^{1 / 2} \delta_{D}(y)^{\alpha / 2}}{|x-y|^{d+\alpha}} \mathbb{P}_{x}\left(X_{t / 2}^{D} \in B\left(x_{0}, \kappa t^{1 / \alpha}\right)\right) .
\end{aligned}
$$

Applying Lemma 3.6, we arrive at the conclusion of the proposition.

Now we are ready to present the proof for Theorem 3.1

Proof of Theorem 3.1. We first assume that $t \leq T_{0}$. By combining Proposition 3.5 (for $\min \left\{\delta_{D}(x), \delta_{D}(y)\right\} \geq t^{1 / \alpha}$ and $|x-y| \leq t^{1 / \alpha}<2|x-y|$ ) and Proposition 3.7 (for $\max \left\{\delta_{D}(x), \delta_{D}(y)\right\} \geq t^{1 / \alpha}, \min \left\{\delta_{D}(x), \delta_{D}(y)\right\}<t^{1 / \alpha}$ and $\left.|x-y| \leq t^{1 / \alpha}<2|x-y|\right)$, we get the conclusion of Theorem 3.1 for $\max \left\{\delta_{D}(x), \delta_{D}(y)\right\} \geq t^{1 / \alpha}$ and $|x-y| \leq$ $t^{1 / \alpha}<2|x-y|$. Using Proposition 3.3 (for $\max \left\{\delta_{D}(x), \delta_{D}(y)\right\} \geq t^{1 / \alpha} \geq 2|x-y|$ ) and the above, we get the conclusion of Theorem 3.1 when

$$
\max \left\{\delta_{D}(x), \delta_{D}(y)\right\} \geq t^{1 / \alpha} \geq|x-y| .
$$

By symmetry, Proposition 3.7 covers the case when $\max \left\{\delta_{D}(x), \delta_{D}(y),|x-y|\right\} \leq t^{1 / \alpha}$.

Now we consider the case $|x-y| \geq t^{1 / \alpha}$. Combining Propositions 3.8 and 3.9 and using symmetry, we obtain the conclusion of Theorem 3.1 for $\min \left\{\delta_{D}(x), \delta_{D}(y)\right\} \leq$ $(t / 2)^{1 / \alpha}$ and $|x-y| \geq t^{1 / \alpha}$. Proposition 3.5 covers the remaining case that $\min \left\{\delta_{D}(x), \delta_{D}(y)\right\} \geq(t / 2)^{1 / \alpha}$ and $|x-y| \geq t^{1 / \alpha}$. We have deduced the conclusion of Theorem 3.1 for $t \leq T_{0}$.

To get it for $T>T_{0}$, it is enough to handle the case $T=2 T_{0}$. Recall that $T_{0}=$ $\left(r_{0} / 16\right)^{\alpha}$. For $(t, x, y) \in\left(T_{0}, 2 T_{0}\right] \times D \times D$, let $x_{0}, y_{0} \in D$ be such that $\max \left\{\left|x-x_{0}\right|\right.$, $\left.\left|y-y_{0}\right|\right\}<r_{0}$ and $\min \left\{\delta_{D}\left(x_{0}\right), \delta_{D}\left(y_{0}\right)\right\} \geq r_{0} / 2$. Then

$$
\begin{aligned}
& (t / 3)^{-d / \alpha} \wedge \frac{t / 3}{|x-z|^{d+\alpha}} \geq c_{1}\left((t / 12)^{-d / \alpha} \wedge \frac{t / 12}{\left|x_{0}-z\right|^{d+\alpha}}\right), \quad z \in D, \\
& (t / 3)^{-d / \alpha} \wedge \frac{t / 3}{|w-y|^{d+\alpha}} \geq c_{1}\left((t / 12)^{-d / \alpha} \wedge \frac{t / 12}{\left|w-y_{0}\right|^{d+\alpha}}\right), \quad w \in D
\end{aligned}
$$

Similarly, there is a positive constant $c_{2}$ such that

$$
(t / 2)^{-d / \alpha} \wedge \frac{t / 2}{\left|x_{0}-y_{0}\right|^{d+\alpha}} \geq c_{2}\left(t^{-d / \alpha} \wedge \frac{t}{|x-y|^{d+\alpha}}\right) .
$$

Let $D_{1}:=\left\{z \in D: \delta_{D}(z)>r_{0} / 4\right\}$. Clearly, $x_{0}, y_{0} \in D_{1}$ and

$$
\min \left\{\delta_{D_{1}}\left(x_{0}\right), \delta_{D_{1}}\left(y_{0}\right)\right\} \geq r_{0} / 4=4\left(T_{0}\right)^{1 / \alpha} \geq 4(t / 2)^{1 / \alpha} .
$$


Combining this with the three displays above and the lower bound estimate in Theorem 3.1 for $p_{D}$ on $\left(0, T_{0}\right] \times D \times D$, we have

$$
\begin{aligned}
p_{D}(t, & x, y)=\int_{D \times D} p_{D}(t / 3, x, z) p_{D}(t / 3, z, w) p_{D}(t / 3, w, y) d z d w \\
\geq & c_{3}\left(1 \wedge \frac{\delta_{D}(x)^{\alpha / 2}}{\sqrt{t / 3}}\right)\left(1 \wedge \frac{\delta_{D}(y)^{\alpha / 2}}{\sqrt{t / 3}}\right) \\
& \cdot \int_{D \times D}\left((t / 3)^{-d / \alpha} \wedge \frac{t / 3}{|x-z|^{d+\alpha}}\right)\left(1 \wedge \frac{\delta_{D}(z)^{\alpha / 2}}{\sqrt{t / 3}}\right) p_{D}(t / 3, z, w) \\
\geq & c_{4}\left(1 \wedge \frac{\delta_{D}(x)^{\alpha / 2}}{\sqrt{t}}\right)\left(1 \wedge \frac{\delta_{D}(y)^{\alpha / 2}}{\sqrt{t}}\right) \int_{D_{1} \times D_{1}}\left((t / 12)^{-d / \alpha} \wedge \frac{t / 3}{\left|x_{0}-z\right|^{d+\alpha}}\right)\left(1 \wedge \frac{\delta_{D}(w)^{\alpha / 2}}{\sqrt{t / 3}}\right) d z d w \\
\geq & c_{5}\left(1 \wedge \frac{\delta_{D}(x)^{\alpha / 2}}{\sqrt{t}}\right)\left(1 \wedge \frac{\delta_{D}(y)^{\alpha / 2}}{\sqrt{t}}\right) \\
& \cdot \int_{D_{1} \times D_{1}} p_{D_{1}}\left(t / 12, x_{0}, z\right) p_{D_{1}}(t / 3, z, w)\left((t / 12)^{-d / \alpha} \wedge \frac{p_{D_{1}}\left(t / 12, w, y_{0}\right) d z d w}{\left|w-y_{0}\right|^{d+\alpha}}\right) d z d w \\
= & c_{5}\left(1 \wedge \frac{\delta_{D}(x)^{\alpha / 2}}{\sqrt{t}}\right)\left(1 \wedge \frac{\delta_{D}(y)^{\alpha / 2}}{\sqrt{t}}\right) p_{D_{1}}\left(t / 2, x_{0}, y_{0}\right) \\
\geq & c_{6}\left(1 \wedge \frac{\delta_{D}(x)^{\alpha / 2}}{\sqrt{t}}\right)\left(1 \wedge \frac{\delta_{D}(y)^{\alpha / 2}}{\sqrt{t}}\right)\left((t / 2)^{-d / \alpha} \wedge \frac{t / 2}{\left|x_{0}-y_{0}\right|^{d+\alpha}}\right) \\
& c_{7}\left(1 \wedge \frac{\delta_{D}(x)^{\alpha / 2}}{\sqrt{t}}\right)\left(1 \wedge \frac{\delta_{D}(y)^{\alpha / 2}}{\sqrt{t}}\right)\left(t^{-d / \alpha} \wedge \frac{t}{|x-y|^{d+\alpha}}\right)
\end{aligned}
$$

for some positive constants $c_{i}, i=3, \ldots, 7$. Here 2.6 is used in the third inequality, and the combination of Propositions 3.3 and 3.5 . which works for any open set and $t>0$, is used in the fourth inequality in view of (3.14). Iterating the above argument one can deduce that the conclusion of Theorem 3.1 holds for $T=k T_{0}$ for any integer $k \geq 2$. This completes the proof of the theorem.

\section{Large time heat kernel estimates and Green function estimates}

In this section, we prove Theorem 1.1 (ii) and Corollary 1.2

Proof of Theorem 1.1 ii). For any bounded $C^{1,1}$ open set $D$ in $\mathbb{R}^{d}$, since $\phi_{D}=e^{\lambda_{1}} P_{1}^{D} \phi_{D}$, we see from Theorem 1.1 i) that on $D$,

$$
\phi_{D}(x) \asymp\left(1 \wedge \delta_{D}(x)^{\alpha / 2}\right) \int_{D}\left(1 \wedge \delta_{D}(y)^{\alpha / 2}\right)\left(1 \wedge \frac{1}{|x-y|^{d+\alpha}}\right) \phi_{D}(y) d y \asymp \delta_{D}(x)^{\alpha / 2} .
$$


This together with (3.7) implies that for any bounded $C^{1,1}$ open set $D$, we have

$$
\begin{aligned}
c_{t}^{-1} \delta_{D}(x)^{\alpha / 2} \delta_{D}(y)^{\alpha / 2} & \leq p_{D}(t, x, y) \\
& \leq c_{t} \delta_{D}(x)^{\alpha / 2} \delta_{D}(y)^{\alpha / 2} \quad \text { for }(t, x, y) \in(0, \infty) \times D \times D .
\end{aligned}
$$

Furthermore, by (3.7), (3.8) and (4.1), there exist $c_{1}>1$ and $T_{1}>0$ such that for all $(t, x, y) \in\left[T_{1}, \infty\right) \times D \times D$,

$$
c_{1}^{-1} e^{-\lambda_{1} t} \delta_{D}(x)^{\alpha / 2} \delta_{D}(y)^{\alpha / 2} \leq p_{D}(t, x, y) \leq c_{1} e^{-\lambda_{1} t} \delta_{D}(x)^{\alpha / 2} \delta_{D}(y)^{\alpha / 2} .
$$

If $T<T_{1}$, by Theorem 1.1 (i), there is a constant $c_{2} \geq 1$ such that

$$
\begin{aligned}
c_{2}^{-1} \delta_{D}(x)^{\alpha / 2} \delta_{D}(y)^{\alpha / 2} & \leq p_{D}(t, x, y) \\
& \leq c_{2} \delta_{D}(x)^{\alpha / 2} \delta_{D}(y)^{\alpha / 2} \quad \text { for } t \in\left[T, T_{1}\right) \text { and } x, y \in D .
\end{aligned}
$$

This establishes Theorem 1.1 ii).

Proof of Corollary 1.2 Put $T:=\operatorname{diam}(D)^{\alpha}$, where $\operatorname{diam}(D)$ is the diameter of $D$. By a change of variable $u=|x-y|^{\alpha} / t$, we have

$$
\begin{aligned}
& \int_{0}^{T}\left(1 \wedge \frac{\delta_{D}(x)^{\alpha / 2}}{\sqrt{t}}\right)\left(1 \wedge \frac{\delta_{D}(y)^{\alpha / 2}}{\sqrt{t}}\right)\left(t^{-d / \alpha} \wedge \frac{t}{|x-y|^{d+\alpha}}\right) d t \\
& =\frac{1}{|x-y|^{d-\alpha}} \int_{|x-y|^{\alpha} / T}^{\infty}\left(u^{d / \alpha-2} \wedge u^{-3}\right)\left(1 \wedge \frac{\sqrt{u} \delta_{D}(x)^{\alpha / 2}}{|x-y|^{\alpha / 2}}\right)\left(1 \wedge \frac{\sqrt{u} \delta_{D}(y)^{\alpha / 2}}{|x-y|^{\alpha / 2}}\right) d u .
\end{aligned}
$$

Note that

$$
\begin{aligned}
\frac{1}{|x-y|^{d-\alpha}} & \int_{1}^{\infty}\left(u^{d / \alpha-2} \wedge u^{-3}\right)\left(1 \wedge \frac{\sqrt{u} \delta_{D}(x)^{\alpha / 2}}{|x-y|^{\alpha / 2}}\right)\left(1 \wedge \frac{\sqrt{u} \delta_{D}(y)^{\alpha / 2}}{|x-y|^{\alpha / 2}}\right) d u \\
& \geq \frac{1}{|x-y|^{d-\alpha}} \int_{1}^{\infty} u^{-3}\left(1 \wedge \frac{\delta_{D}(x)^{\alpha / 2}}{|x-y|^{\alpha / 2}}\right)\left(1 \wedge \frac{\delta_{D}(y)^{\alpha / 2}}{|x-y|^{\alpha / 2}}\right) d u \\
& =\frac{1}{2|x-y|^{d-\alpha}}\left(1 \wedge \frac{\delta_{D}(x)^{\alpha / 2}}{|x-y|^{\alpha / 2}}\right)\left(1 \wedge \frac{\delta_{D}(y)^{\alpha / 2}}{|x-y|^{\alpha / 2}}\right)
\end{aligned}
$$

while

$$
\begin{aligned}
& \frac{1}{|x-y|^{d-\alpha}} \int_{1}^{\infty}\left(u^{d / \alpha-2} \wedge u^{-3}\right)\left(1 \wedge \frac{\sqrt{u} \delta_{D}(x)^{\alpha / 2}}{|x-y|^{\alpha / 2}}\right)\left(1 \wedge \frac{\sqrt{u} \delta_{D}(y)^{\alpha / 2}}{|x-y|^{\alpha / 2}}\right) d u \\
& =\frac{1}{|x-y|^{d-\alpha}} \int_{1}^{\infty} u^{-2}\left(u^{-1 / 2} \wedge \frac{\delta_{D}(x)^{\alpha / 2}}{|x-y|^{\alpha / 2}}\right)\left(u^{-1 / 2} \wedge \frac{\delta_{D}(y)^{\alpha / 2}}{|x-y|^{\alpha / 2}}\right) d u \\
& \quad \leq \frac{1}{|x-y|^{d-\alpha}} \int_{1}^{\infty} u^{-2}\left(1 \wedge \frac{\delta_{D}(x)^{\alpha / 2}}{|x-y|^{\alpha / 2}}\right)\left(1 \wedge \frac{\delta_{D}(y)^{\alpha / 2}}{|x-y|^{\alpha / 2}}\right) d u \\
& =\frac{1}{|x-y|^{d-\alpha}}\left(1 \wedge \frac{\delta_{D}(x)^{\alpha / 2}}{|x-y|^{\alpha / 2}}\right)\left(1 \wedge \frac{\delta_{D}(y)^{\alpha / 2}}{|x-y|^{\alpha / 2}}\right) .
\end{aligned}
$$


By Theorem 1.1.ii),

$$
\int_{T}^{\infty} p_{D}(t, x, y) \asymp \delta_{D}(x)^{\alpha / 2} \delta_{D}(y)^{\alpha / 2}
$$

(i) Assume $d>\alpha$. Observe that

$$
\begin{aligned}
\frac{1}{|x-y|^{d-\alpha}} & \int_{|x-y|^{\alpha} / T}^{1}\left(u^{d / \alpha-2} \wedge u^{-3}\right)\left(1 \wedge \frac{\sqrt{u} \delta_{D}(x)^{\alpha / 2}}{|x-y|^{\alpha / 2}}\right)\left(1 \wedge \frac{\sqrt{u} \delta_{D}(y)^{\alpha / 2}}{|x-y|^{\alpha / 2}}\right) d u \\
& \leq \frac{1}{|x-y|^{d-\alpha}}\left(1 \wedge \frac{\delta_{D}(x)^{\alpha / 2}}{|x-y|^{\alpha / 2}}\right)\left(1 \wedge \frac{\delta_{D}(y)^{\alpha / 2}}{|x-y|^{\alpha / 2}}\right) \int_{0}^{1} u^{d / \alpha-2} d u \\
& \leq \frac{\alpha}{d-\alpha} \frac{1}{|x-y|^{d-\alpha}}\left(1 \wedge \frac{\delta_{D}(x)^{\alpha / 2}}{|x-y|^{\alpha / 2}}\right)\left(1 \wedge \frac{\delta_{D}(y)^{\alpha / 2}}{|x-y|^{\alpha / 2}}\right)
\end{aligned}
$$

So by Theorem 1.1 and 4.2 -4.6 , we have

$$
\begin{aligned}
G_{D}(x, y) & =\int_{0}^{T} p_{D}(t, x, y) d t+\int_{T}^{\infty} p_{D}(t, x, y) d t \\
& \asymp \frac{1}{|x-y|^{d-\alpha}}\left(1 \wedge \frac{\delta_{D}(x)^{\alpha / 2}}{|x-y|^{\alpha / 2}}\right)\left(1 \wedge \frac{\delta_{D}(y)^{\alpha / 2}}{|x-y|^{\alpha / 2}}\right)+\delta_{D}(x)^{\alpha / 2} \delta_{D}(y)^{\alpha / 2} \\
& \asymp \frac{1}{|x-y|^{d-\alpha}}\left(1 \wedge \frac{\delta_{D}(x)^{\alpha / 2}}{|x-y|^{\alpha / 2}}\right)\left(1 \wedge \frac{\delta_{D}(y)^{\alpha / 2}}{|x-y|^{\alpha / 2}}\right) .
\end{aligned}
$$

In the last estimate, we used the fact that $D$ is bounded. Since $\delta_{D}(x) \leq \delta_{D}(y)+|x-y|$ for every $x, y \in D$, it is easy to see that for every $r \in(0,1]$,

$$
\left(1 \wedge \frac{r \delta_{D}(x)}{|x-y|}\right)\left(1 \wedge \frac{r \delta_{D}(y)}{|x-y|}\right) \leq 1 \wedge \frac{r^{2} \delta_{D}(x) \delta_{D}(y)}{|x-y|^{2}} \leq 2\left(1 \wedge \frac{r \delta_{D}(x)}{|x-y|}\right)\left(1 \wedge \frac{r \delta_{D}(y)}{|x-y|}\right) .
$$

So on $D \times D$,

$$
G_{D}(x, y) \asymp \frac{1}{|x-y|^{d-\alpha}}\left(1 \wedge \frac{\delta_{D}(x)^{\alpha / 2} \delta_{D}(y)^{\alpha / 2}}{|x-y|^{\alpha}}\right) .
$$

For the other cases, we let

$$
u_{0}:=\frac{\delta_{D}(x)^{\alpha / 2} \delta_{D}(y)^{\alpha / 2}}{|x-y|^{\alpha}}
$$

Clearly $1 / u_{0} \geq|x-y|^{\alpha} / \operatorname{diam}(D)^{\alpha}=|x-y|^{\alpha} / T$. 
(ii) Now assume $d=\alpha=1$. We have

$$
\begin{aligned}
\frac{1}{|x-y|^{d-\alpha}} \int_{|x-y|^{\alpha} / T}^{1} & \left(u^{d / \alpha-2} \wedge u^{-3}\right)\left(1 \wedge \frac{\sqrt{u} \delta_{D}(x)^{\alpha / 2}}{|x-y|^{\alpha / 2}}\right)\left(1 \wedge \frac{\sqrt{u} \delta_{D}(y)^{\alpha / 2}}{|x-y|^{\alpha / 2}}\right) d u \\
& \asymp \int_{|x-y|^{\alpha} / T}^{1} u^{-1}\left(1 \wedge \frac{u \delta_{D}(x)^{\alpha / 2} \delta_{D}(y)^{\alpha / 2}}{|x-y|^{\alpha}}\right) d u \\
= & \int_{|x-y|^{\alpha} / T}^{1} u^{-1} \mathbf{1}_{\left\{u \geq 1 / u_{0}\right\}} d u+\int_{|x-y|^{\alpha} / T}^{1} u_{0} \mathbf{1}_{\left\{u<1 / u_{0}\right\}} d u \\
= & \log \left(u_{0} \vee 1\right)+u_{0}\left(\left(1 / u_{0}\right) \wedge 1-|x-y|^{\alpha} / T\right) .
\end{aligned}
$$

So by Theorem 1.1, 4.2 4.5 and 4.7 -4.9 ,

$$
\begin{aligned}
G_{D}(x, y)= & \int_{0}^{T} p_{D}(t, x, y) d t+\int_{T}^{\infty} p_{D}(t, x, y) d t \\
\asymp & \left(1 \wedge \frac{\delta_{D}(x)^{\alpha / 2}}{|x-y|^{\alpha / 2}}\right)\left(1 \wedge \frac{\delta_{D}(y)^{\alpha / 2}}{|x-y|^{\alpha / 2}}\right)+\log \left(u_{0} \vee 1\right) \\
& +u_{0}\left(\left(1 / u_{0}\right) \wedge 1-|x-y|^{\alpha} / T\right)+\delta_{D}(x)^{\alpha / 2} \delta_{D}(y)^{\alpha / 2} \\
\asymp & 1 \wedge u_{0}+\log \left(u_{0} \vee 1\right)+u_{0}\left(\left(1 / u_{0}\right) \wedge 1-|x-y|^{\alpha} / T\right)+\delta_{D}(x)^{\alpha / 2} \delta_{D}(y)^{\alpha / 2} \\
\asymp & 1 \wedge u_{0}+\log \left(u_{0} \vee 1\right)+\delta_{D}(x)^{\alpha / 2} \delta_{D}(y)^{\alpha / 2} \\
\asymp & \log \left(1+u_{0}\right)+\delta_{D}(x)^{\alpha / 2} \delta_{D}(y)^{\alpha / 2} \\
\asymp & \log \left(1+\frac{\delta_{D}(x)^{\alpha / 2} \delta_{D}(y)^{\alpha / 2}}{|x-y|^{\alpha}}\right) .
\end{aligned}
$$

In the last estimate, we used the fact that $D$ is bounded.

(iii) Lastly we consider the case $d=1<\alpha<2$. By 4.7)-4.8,

$$
\begin{aligned}
& \frac{1}{|x-y|^{d-\alpha}} \int_{|x-y|^{\alpha} / T}^{1}\left(u^{d / \alpha-2} \wedge u^{-3}\right)\left(1 \wedge \frac{\sqrt{u} \delta_{D}(x)^{\alpha / 2}}{|x-y|^{\alpha / 2}}\right)\left(1 \wedge \frac{\sqrt{u} \delta_{D}(y)^{\alpha / 2}}{|x-y|^{\alpha / 2}}\right) d u \\
& \asymp \frac{1}{|x-y|^{1-\alpha}} \int_{|x-y|^{\alpha} / T}^{1} u^{1 / \alpha-2}\left(1 \wedge \frac{u \delta_{D}(x)^{\alpha / 2} \delta_{D}(y)^{\alpha / 2}}{|x-y|^{\alpha}}\right) d u \\
& =\frac{1}{|x-y|^{1-\alpha}}\left(\int_{|x-y|^{\alpha} / T}^{1} u^{1 / \alpha-2} \mathbf{1}_{\left\{u \geq 1 / u_{0}\right\}} d u+\int_{|x-y|^{\alpha} / T}^{1} u_{0} u^{1 / \alpha-1} \mathbf{1}_{\left\{u<1 / u_{0}\right\}} d u\right) \\
& =\frac{1}{|x-y|^{1-\alpha}}\left(\frac{\alpha}{\alpha-1}\left(\left(u_{0} \vee 1\right)^{1-1 / \alpha}-1\right)+\alpha u_{0}\left(\left(u_{0} \vee 1\right)^{-1 / \alpha}-\left(|x-y|^{\alpha} / T\right)^{1 / \alpha}\right)\right) .
\end{aligned}
$$


So by Theorem 1.1, 4.2)-4.5, (4.7)-4.8 and the last display, we have

$$
\begin{aligned}
& G_{D}(x, y)=\int_{T}^{\infty} p_{D}(t, x, y) d t+\int_{0}^{T} p_{D}(t, x, y) d t \\
& \asymp \delta_{D}(x)^{\alpha / 2} \delta_{D}(y)^{\alpha / 2}+\frac{1}{|x-y|^{1-\alpha}}\left(1 \wedge u_{0}\right) \\
& \quad+\frac{1}{|x-y|^{1-\alpha}}\left(\left(\left(u_{0} \vee 1\right)^{1-1 / \alpha}-1\right)+u_{0}\left(\left(u_{0} \vee 1\right)^{-1 / \alpha}-\left(|x-y|^{\alpha} / T\right)^{1 / \alpha}\right)\right) \\
& \asymp \delta_{D}(x)^{\alpha / 2} \delta_{D}(y)^{\alpha / 2}+\frac{1}{|x-y|^{1-\alpha}}\left(u_{0} \wedge u_{0}^{1-1 / \alpha}\right) \\
& =\delta_{D}(x)^{\alpha / 2} \delta_{D}(y)^{\alpha / 2}+\frac{1}{|x-y|^{1-\alpha}}\left(\frac{\delta_{D}(x)^{\alpha / 2} \delta_{D}(y)^{\alpha / 2}}{|x-y|^{\alpha}} \wedge \frac{\delta_{D}(x)^{(\alpha-1) / 2} \delta_{D}(y)^{(\alpha-1) / 2}}{|x-y|^{\alpha-1}}\right) \\
& \asymp\left(\delta_{D}(x) \delta_{D}(y)\right)^{(\alpha-1) / 2} \wedge \frac{\delta_{D}(x)^{\alpha / 2} \delta_{D}(y)^{\alpha / 2}}{|x-y|} .
\end{aligned}
$$

In the last estimate, we used the fact that $D$ is bounded. This proves the corollary.

Acknowledgments. We thank the two referees for their helpful comments on the first version of this paper.

Research of Z.-Q. Chen was partially supported by NSF Grant DMS-0600206.

Research of P. Kim was supported by Basic Science Research Program through the National Research Foundation of Korea (NFR) grant funded by the Korea government (MEST)(20100001984).

\section{References}

[1] Blumenthal, R. M., Getoor, R. K.: Some theorems on stable processes. Trans. Amer. Math. Soc. 95, 263-273 (1960) Zbl 0107.12401 MR 0119247

[2] Bogdan, K.: The boundary Harnack principle for the fractional Laplacian. Studia Math. 123, 43-80 (1997) Zbl 0870.31009 MR 1438304

[3] Caffarelli, L. A., Salsa, S., Silvestre, L.: Regularity estimates for the solution and the free boundary to the obstacle problem for the fractional Laplacian. Invent. Math. 171, 425-461 (2008) Zbl 1148.35097 MR 2367025

[4] Carlen, E. A., Kusuoka, S., Stroock, D. W.: Upper bounds for symmetric Markov transition functions. Ann. Inst. H. Poincaré 23, 245-287 (1987) Zbl 0634.60066 MR 0898496

[5] Chen, Z.-Q., Kim, P., Kumagai, T.: Weighted Poincaré inequality and heat kernel estimates for finite range jump processes. Math. Ann. 342, 833-883 (2008) Zbl $1156.60069 \mid$ MR 2443765

[6] Chen, Z.-Q., Kim, P., Song, R.: Two-sided heat kernel estimates for censored stable-like processes. Probab. Theory Related Fields 146, 361-399 (2010) MR 2574732

[7] Chen, Z.-Q., Kim, P., Song, R.: Sharp heat kernel estimates for relativistic stable processes in open sets. Preprint

[8] Chen, Z.-Q., Kumagai, T.: Heat kernel estimates for stable-like processes on $d$-sets. Stoch. Process. Appl. 108, 27-62 (2003) Zbl 1075.60556 MR 2008600

[9] Chen, Z.-Q., Kumagai, T.: Heat kernel estimates for jump processes of mixed types on metric measure spaces. Probab. Theory Related Fields 140, 277-317 (2008) Zbl 1131.60076 MR 2357678 
[10] Chen, Z.-Q., Song, R.: Intrinsic ultracontractivity and conditional gauge for symmetric stable processes. J. Funct. Anal. 150, 204-239 (1997) Zbl 0886.60072 MR 1473631

[11] Chen, Z.-Q., Song, R.: Estimates on Green functions and Poisson kernels for symmetric stable processes. Math. Ann. 312, 465-501 (1998) Zbl 0918.60068 MR 1654824

[12] Davies, E. B.: Explicit constants for Gaussian upper bounds on heat kernels. Amer. J. Math. 109, 319-333 (1987) Zbl 0659.35009

[13] Davies, E. B.: The equivalence of certain heat kernel and Green function bounds. J. Funct. Anal. 71, 88-103 (1987) Zbl 0652.35023 MR 0879702

[14] Davies, E. B.: Heat Kernels and Spectral Theory. Cambridge Univ. Press, Cambridge (1989) Zbl 0699.35006 MR 0990239

[15] Davies, E. B., Simon, B.: Ultracontractivity and the heat kernel for Schrödinger operators and Dirichlet Laplacians. J. Funct. Anal. 59, 335-395 (1984) Zbl 0568.47034 MR 0766493

[16] Fabes, E. B., Garofalo, N., Salsa, S.: A backward Harnack inequality and Fatou theorem for nonnegative solutions of parabolic equations. Illinois J. Math. 30, 536-565 (1986) Zbl 0625.35006 MR 0857210

[17] Getoor, R. K.: First passage times for symmetric stable processes in space. Trans. Amer. Math. Soc. 101, 75-90 (1961) Zbl 0104.11203 MR 0137148

[18] Gilbarg, D., Trudinger, N. S.: Elliptic Partial Differential Equations of Second Order. 2nd ed., Springer (1983) Zbl 0562.35001 MR 0737190

[19] Ikeda, N., Watanabe, S.: Stochastic Differential Equations and Diffusion Processes. 2nd ed., North-Holland (1989) Zbl 0684.60040 MR 1011252

[20] Janicki, A., Weron, A.: Simulation and Chaotic Behavior of $\alpha$-Stable Stochastic Processes. Dekker (1994) Zbl 0946.60028 MR 1306279

[21] Kulczycki, T.: Properties of Green function of symmetric stable processes, Probab. Math. Statist. 17, 339-364 (1997) Zbl 0903.60063 MR 1490808

[22] Kulczycki, T.: Intrinsic ultracontractivity for symmetric stable processes. Bull. Polish Acad. Sci. Math. 46, 325-334 (1998) Zbl 0917.60071 MR 1643611

[23] Kulczycki, T., Siudeja, B.: Intrinsic ultracontractivity of the Feynman-Kac semigroup for relativisitic stable processes. Trans. Amer. Math. Soc. 358, 5025-5057 (2006) Zbl 1112.47034 MR 2231884

[24] Samorodnitsky, G., Taqqu, M. S.: Stable Non-Gaussian Random Processes. Chapman \& Hall, New York-London (1994) Zbl 0925.60027 MR 1280932

[25] Silvestre, L.: Hölder estimates for solutions of integro-differential equations like the fractional Laplace. Indiana Univ. Math. J. 55, 1155-1174 (2006) Zbl 1101.45004 MR 2244602

[26] Siudeja, B.: Symmetric stable processes on unbounded domains. Potential Anal. 25, 371-386 (2006) Zbl 1111.31004 MR 2255353

[27] Zhang, Q. S.: The boundary behavior of heat kernels of Dirichlet Laplacians. J. Differential Equations 182, 416-430 (2002) Zbl 1002.35052 MR 1900329 